\title{
Genetic variations in vitamin D-related pathways and breast cancer risk in African American women in the AMBER consortium
}

\section{Song Yao ${ }^{1}$, Stephen A. Haddad ${ }^{2}$, Qiang Hu${ }^{3}$, Song Liu ${ }^{3}$, Kathryn L. Lunetta ${ }^{4}$, Edward A. Ruiz-Narvaez ${ }^{2}$, Chi-Chen Hong ${ }^{1}$, Qianqian Zhu ${ }^{3}$, Lara Sucheston-Campbell ${ }^{1}$, Ting-Yuan David Cheng $^{1}$, Jeannette T. Bensen ${ }^{5}$, Candace S. Johnson ${ }^{6}$, Donald L. Trump ${ }^{7}$, Christopher}

A. Haiman ${ }^{8}$, Andrew F. Olshan ${ }^{5}$, Julie R. Palmer ${ }^{2}$, and Christine B. Ambrosone ${ }^{1}$

${ }^{1}$ Department of Cancer Prevention and Control, Roswell Park Cancer Institute, Buffalo, NY

${ }^{2}$ Slone Epidemiology Center at Boston University, Boston, MA

${ }^{3}$ Department of Biostatistics and Bioinformatics, Roswell Park Cancer Institute, Buffalo, NY

${ }^{4}$ Department of Biostatistics, Boston University School of Public Health, Boston, MA

${ }^{5}$ Department of Epidemiology, Gillings School of Global Public Health, University of North

Carolina at Chapel Hill, Chapel Hill, NC

${ }^{6}$ Department of Pharmacology and Therapeutics, Roswell Park Cancer Institute, Buffalo, NY

${ }^{7}$ Inova Schar Cancer Institute, Falls Church, VA

${ }^{8}$ Department of Preventive Medicine, Keck School of Medicine, University of Southern California/ Norris Comprehensive Cancer Center, Los Angeles, CA

\section{Abstract}

Studies of genetic variations in vitamin D-related pathways and breast cancer risk have been conducted mostly in populations of European ancestry, and only sparsely in African Americans (AA), who are known for a high prevalence of vitamin D deficiency. We analyzed 24,445 germline variants in 63 genes from vitamin D-related pathways in the African American Breast Cancer Epidemiology and Risk (AMBER) consortium, including 3,663 breast cancer cases and 4,687 controls. Odds ratios (OR) were derived from logistic regression models for overall breast cancer, by estrogen receptor (ER) status (1,983 ER positive and 1,098 ER negative), and for case-only analyses of ER status. None of the three vitamin D-related pathways were associated with breast cancer risk overall or by ER status. Gene-level analyses identified associations with risk for several genes at a nominal $p \leq 0.05$, particularly for ER- breast cancer, including rs4647707 in $D D B 2$. In case-only analyses, vitamin D metabolism and signaling pathways were associated with ER-cancer (pathway-level $p=0.02$ ), driven by a single gene $C A S R$ (gene-level $p=0.001$ ). The top SNP in $C A S R$ was rs112594756 ( $p=7 \times 10^{-5}$, gene-wide corrected $\left.p=0.01\right)$, followed by a second signal from a nearby SNP rs6799828 $\left(p=1 \times 10^{-4}\right.$, corrected $\left.p=0.03\right)$. In summary,

Correspondence to: Song Yao, Department of Cancer Prevention and Control, Roswell Park Cancer Institute, Elm \& Carlton Streets, Buffalo, NY 14263, Tel: 716-845-4968; Fax: 1-716-845-1356, song.yao@ roswellpark.org.

Additional Supporting Information may be found in the online version of this article. 
several variants in vitamin D pathways were associated with breast cancer risk in AA women. In addition, $C A S R$ may be related to tumor ER status, supporting a role of vitamin $\mathrm{D}$ or calcium in modifying breast cancer phenotypes.

\section{Keywords}

vitamin D; breast cancer; genetic variation; African American; estrogen receptor

Vitamin D plays a central role in skeletal development and maintenance. A primary source of vitamin D in humans is cutaneous synthesis under sunlight exposure. Ultraviolet-B (UVB) light converts 7-dehydrocholesterol to vitamin D precursor, which undergoes a cascade of further enzymatic reactions until fully activated. The active vitamin D metabolite, 1a,25-dihydroxyvitamin $\mathrm{D}$, acts by binding to the vitamin $\mathrm{D}$ receptor (VDR), altering transcription of many target genes.

Over the last two decades, many extra-skeletal effects of vitamin D have been delineated, including a series of anti-cancer activities, from anti-proliferation to induction of apoptosis, promotion of differentiation, anti-inflammation, and inhibition of angiogenesis and metastasis. ${ }^{1,2}$ These findings support the biologic basis for a relationship of vitamin D deficiency with cancer morbidity and mortality, complementing the ecological evidence. ${ }^{3}$

There is no definitive evidence regarding the role of vitamin D in breast cancer prevention. Epidemiologic studies of sun exposure, dietary and supplementary vitamin D intake, and circulating vitamin $\mathrm{D}$ biomarkers, provide inconclusive results. ${ }^{4-6} \mathrm{~A}$ candidate gene approach has also been used to study genetic variations with breast cancer risk, with no consistent findings across studies. ${ }^{7-9}$ These previous studies were based largely on populations of European ancestry (EA) and focused on only a small number of markers and genes.

AAs have a high rate of vitamin D deficiency, likely due, in part, to dark skin pigmentation which blocks UVB light, and, to a lesser extent, lactose intolerance which may limit vitamin D intake from fortified dairy products. ${ }^{10}$ Our previous studies have also demonstrated widespread differences in variant frequency and linkage disequilibrium (LD) in vitamin D-related genes between AA and EA populations. ${ }^{11}$ It is thus important to understand whether ancestral variations in vitamin D-related genes in AAs, which were shaped over millennia in sun exposure-abundant Africa, would put AA women at high risk of breast cancer in a Northern hemisphere environment where sun exposure is limited and seasonally fluctuates. ${ }^{10}$

Associations may also differ according to estrogen receptor (ER) status, given the increasing recognition of breast cancer etiological heterogeneity. ${ }^{12}$ In an earlier study, we found that circulating levels of $25(\mathrm{OH})$ vitamin D were inversely associated with aggressive breast cancer characteristics, including ER negative (ER-) tumors. ${ }^{13}$ Because AA women are more likely to be diagnosed with ER- cancer than EA women and have a higher prevalence of vitamin D deficiency, it is plausible to extrapolate that genetic variations in vitamin Drelated pathways may be associated with ER status in AA women. In this study, we 
comprehensively examined genetic variations in vitamin D-related pathways with breast cancer risk in a large AA breast cancer consortium.

\section{Study Population and Methods}

\section{The African American Breast Cancer Etiology and Risk (AMBER) consortium}

The AMBER consortium was established in 2011 to integrate epidemiologic resources of four existing studies with the primary goal to aggregate an adequate sample size to study epidemiology of breast cancer subtypes in AA women. The four studies include two casecontrol studies, the Women's Circle of Health Study (WCHS) and the Carolina Breast Cancer Study (CBCS), and two prospective cohort studies, the Black Women's Health Study (BWHS) and the Multi-Ethnic Cohort (MEC). A detailed description of the consortium and the four contributing studies can be found elsewhere. ${ }^{14-19}$

The WCHS is a case-control study enrolling breast cancer patients of age 25-75 years, initially in New York City (NYC) and New Jersey (NJ), and later exclusively in NJ. ${ }^{16,17}$ The enrollment began in 2002 with incident breast cancer patients ascertained and consented in NYC hospitals with large referral patterns of AAs and through the NJ State Cancer Registry. Controls matched on state, race and age were recruited from random digital dialing and community events. The CBCS is a population-based case-control study in North Carolina beginning in $1993 .{ }^{15}$ Breast cancer patients of age 20-74 were identified through state cancer registry, and controls were enrolled through Division of Motor Vehicle lists and Health Care Finance Administration lists.

The BWHS is a prospective study of 59,000 AA women across the US who were 21-69 years of age at the study entry in 1995 and have been followed by biennial questionnaire since that time. ${ }^{18}$ Women diagnosed with breast cancer are identified by self-report in follow-up questionnaires, and confirmed by medical records, state cancer registries, and the National Death Index. The MEC is a multiethnic prospective cohort in Hawaii and southern California with follow-up of 215,000 men and women aged 45-75 at the time of study entry in $1993 .{ }^{19}$ Breast cancer diagnoses are confirmed by linkage to state cancer registries and the National Death Index. Controls for the BWHS and MEC were identified from among AA participants who had not been diagnosed with breast cancer.

All study participants provided consent for using their data and specimens for research purposes, and the study was approved by Institutional Review Boards at participating institutions. A descriptive summary of the number of cases and controls from each contributing study included in this analysis, with index age and ER status in cases obtained from pathology reports, is provided in Table 1 .

\section{Genetic marker selection, genotyping, quality control and imputation}

For genotyping efforts in the BWHS, CBCS and WCHS, a systematic approach was used to select all known candidate genes from three vitamin D-related pathways defined by the Molecular Signature Database $(\mathrm{MSigDB})^{20}$ : the vitamin D metabolism and signaling pathway, the pigmentation synthesis and metabolism pathway, and the UV exposure response pathway (Table 2). TagSNPs were chosen from each gene using criteria of $r^{2} \geq 0.8$ 
and minor allele frequency $\geq 10 \%$ in the Yoruban (YRI) population from the 1000 Genome Project. $^{21}$ These SNPs were added as part of the custom content to the Illumina Human Exome Beadchip v1.1.

Genotyping was performed by the Center for Inherited Disease Research (CIDR), followed by stringent sample and marker QC steps. ${ }^{22}$ Imputation to the 1000 Genomes data using the IMPUTE2 program ${ }^{23}$ was performed by the University of Washington (UW). MEC samples had been geno-typed previously using the Illumina 1M-Duo chip and also imputed to the 1000 Genomes data. The imputed MEC data were pooled with those from the BWHS, CBCS and WCHS to create a final analytical dataset. Markers with mismatching alleles or allele frequencies that were different by $>0.15$ between MEC and the other three studies were excluded. Also, markers with MAF $<0.6 \%$ or imputation info score- $<0.5$ in either study were removed. For this analysis of vita-min D-related pathways, a total of 24,445 variants in 63 genes were included.

\section{Statistical analysis}

Population structure by principle component analysis (PCA) was assessed by the smartpca program in the EIGENSOFT package. ${ }^{24}$ A plot of the top two principal components (PCs) of the study population with HapMap controls is shown in Supporting Information Figure 1. Relatedness was assessed by PLINK. ${ }^{25}$ Thirty-five individual outliers in PCA and 162 1stdegree relatives identified were flagged for sensitivity analysis. No substantial changes in risk estimates were found after excluding these individuals and they thus were kept in the analysis. Ten PCs were tested with case-control status while controlling for covariates, including index age, study, geographic region and DNA source, and none was significantly associated with breast cancer risk. To be conservative, three PCs with a $p<0.10$ were included in the logistic regression models.

Breast cancer risk was analyzed overall, and separately for ER positive (+), and ER- disease compared to controls. We also performed case-only analysis, with ER+ cases as the "controls", to assess potential differential associations between genetic variants and ER subgroups. Three levels of analyses of genetic variations were performed: pathway-level, gene-level and single marker-level, under the hypothesis that aggregating the effects of multiple markers within a gene or a biological pathway might be more statistically powerful and less prone to multiple testing bias than single marker analysis. Pathway- and gene-level analyses were performed first, using the adaptive rank truncated product (ARTP) statistic, ${ }^{26}$ which can optimize the number of single marker $p$-values combined in each gene-level and pathway-level test. For pathway-level analysis, the PIGE software implementation of the ARTP method takes gene-level information into consideration when combining markers in a pathway (https://cran.r-project.org/web/packages/PIGE/index.html). To avoid redundancy of markers in high LD $\left(r^{2} \geq 0.8\right)$, the ARTP gene-level tests combined the optimal number of most significant SNP $p$-values from among the top 10 pruned-in SNPs for each gene. The ARTP pathway tests combined the optimal percentage (in 5\% increments) of the most significant gene $p$-values in each pathway, without exceeding $50 \%$. This parameter of the top 10 pruned markers was chosen to ensure excellent representation of the genetic variations, but also not to dilute any effects from truly causal markers by including too many null 
markers in the analysis. Following gene-level testing, single marker-level analyses were only pursued within genes reaching a nominal significance level of 0.05 using PLINK with dosage data and controlling for age, study, geographic region, DNA source, and three top PCs. We corrected for multiple testing within these genes with a Bonferroni correction for the effective number of independent markers tested within a gene using Gao's SimpleM approach, ${ }^{27}$ and called this the "gene-wide" significance. Single marker associations for top genes were plotted with linkage disequilibrium data using the LocusZoom program. ${ }^{28}$

\section{Results}

At the pathway-level, none of the three vitamin D-related pathways was associated with breast cancer risk overall or stratified by ER status $(p>0.05)$. At the gene-level, several genes were associated with breast cancer risk at nominal $p \leq 0.05$. We considered singlemarker associations only within genes that at the gene-level demonstrated nominal significance at $p \leq 0.05$, and we corrected for multiple comparisons within genes. For overall breast cancer risk, four genes, including $B C L 3, M A P K 8, R E V 1$ and $C Y P 2 R 1$, were identified (Table 2). The most significant gene was $R E V 1(p=0.004)$, which encodes for DNA repair protein REV1 (Fig. 1a). The most significant variant in this gene was an intronic SNP rs9308822, with the $\mathrm{C}$ allele associated with decreased odds of breast cancer (OR $=$ $0.86,95 \% \mathrm{CI}=0.80-0.93, p=1 \times 10^{-4}$, gene-wide corrected $\left.p=0.01\right)$ (Table 3). Two other variants, one each in BCL3 and PAICS, also remained significant at a gene-wide level.

Five genes were associated with ER+ breast cancer, including PAICS, BCL3, ERCC6, $R E V 1$ and $C Y P 2 R 1$ (Table 2), with 5 variants significant at a gene-wide level (Table 3). The top variant was rs114723899 residing in the $3^{\prime}$ UTR of ERCC6 (Fig. 1 b). The $\mathrm{C}$ allele was associated with decreased risk of $\mathrm{ER}+$ cancer $(\mathrm{OR}=0.62,95 \% \mathrm{CI}=0.49-0.78, p=4 \times$ $10^{-5}$, gene-wide corrected $p=0.005$ ). Three genes, including $D C T, S L C 24 A 4$, and $D D B 2$, were associated with ER- breast cancer, with 2 SNPs significant at a gene-wide level. The most significant SNP, rs 4647707 , resides in the $5^{\prime}$ UTR of $D D B 2$, which encodes for DNA damage-binding protein 2 (Fig. 1c). The A allele was associated with increased odds of ERcancer $\left(\mathrm{OR}=1.26,95 \% \mathrm{CI}=1.1321 .41, p=4 \times 10^{-5}\right.$, gene-wide corrected $p=0.003$ ). Based on RegulomeDB, this SNP is likely to affect transcription factor binding.

In case-only analyses, the vitamin D metabolism and signaling pathway was associated with ER-status (ER- vs. ER+) at a nominal pathway-level $p$ values of 0.02 . Among genes in this pathway, $C A S R$, which encodes for calcium sensing receptor, was the only gene significantly associated with ER status $(p=0.001)$ (Table 2$)$ after correction for multiple comparison of 11 genes in the pathway and 4 endpoints. The most significant SNP in CASR was an intronic SNP rs112594756 ( $p=7 \times 10^{-5}$, gene-wide corrected $\left.p=0.01\right)$ (Fig. 1d). The $\mathrm{G}$ allele of this SNP was associated with increased odds of ER- breast cancer relative to $\mathrm{ER}+$ disease $(\mathrm{OR}=1.27,95 \% \mathrm{CI}=1.13-1.43$ (Table 3). A second independent intronic SNP in $C A S R$, rs6799828, was also significantly related to ER status at a gene-wide level ( $p$ $=1 \times 10^{-4}$, corrected $p=0.03$ ), with the $\mathrm{G}$ allele associated with increased odds of ERbreast cancer compared to $\mathrm{ER}+(\mathrm{OR}=1.24,95 \% \mathrm{CI}=1.1121 .39)$. Both SNPs were associated with increased risk of ER-negative cancer but slightly reduced risk of ER-positive cancer in case-control analyses. 
In addition to the vitamin $\mathrm{D}$ metabolism and signaling pathway, four genes from the other two pathways reached nominal significance level in the case-only analyses: $A L A S 2, D C T$, IRF4 and DDB2 (Table 2). One variant in $D C T$ was significant at the gene-wide level (Table $3)$.

\section{Discussion}

In this analysis of data and samples from 3,663 AA women with breast cancer and 4,687 controls, we examined associations between breast cancer risk and variants in three vitamin D-related pathways: (1) pigment synthesis and metabolism, (2) response to UV exposure and (3) vitamin D metabolism and signaling. Although there were no significant associations between any of these pathways and breast cancer risk overall, we did observe that the vitamin D metabolism and signaling pathway was significantly associated with ER- breast cancer relative to ER+ disease. These associations were driven primarily by two independent loci from one gene, $C A S R$. At the gene level, there were also variants that were preferentially associated with risk of ER- $(D D B 2, D C T)$ and ER+ (ERCC6, PAICS, $C Y P 2 R 1, B C L 3$ and $R E V 1$ ) breast cancer, indicating a role for vitamin $\mathrm{D}$ in diverging etiologic pathways for breast cancer subtypes.

Data from experimental studies in cell culture and animal models support a variety of anticancer properties of vitamin $\mathrm{D}$, which, however, have yet to be confirmed in epidemiologic studies and prospective trials. ${ }^{1,2}$ Current literature on the relationship between circulating levels of vitamin D bio-marker, 25-hydroxyvitamin D (25OHD) and breast cancer risk is mixed. ${ }^{4-6}$ Existing studies of vitamin D-related genetic variations also provide inconclusive results. ${ }^{7-9}$ Many of the earlier studies were focused on several commonly studied polymorphisms in vitamin D receptor (VDR), including Fok1 (rs2228570), Apa1 (rs7975232), Bsm1 (rs1544410) and Taq1 (rs731236). Although one recent meta-analysis concluded that the functional Fok1 polymorphism was associated with significantly increased risk, ${ }^{9}$ the effect size was small $(\mathrm{OR}=1.09)$, and has not been detected by large GWAS. Because two GWAS of circulating 25OHD concentrations have identified a few variants, including those in $G C$ and $C Y P 2 R 1$, significant at genome-wide level, ${ }^{29,30}$ a recent study, based on the Breast and Prostate Cancer Cohort Consortium (BPC3) of mainly individuals of European descent, attempted to examine whether those GWAS variants were also associated with breast cancer risk under a hypothesis of Mendelian randomization. ${ }^{31}$ However, results from this study were null.

The lack of strong associations between overall breast cancer risk and vitamin D-related genetic variants in our study may be attributed to etiological heterogeneity, i.e., distinct disease subgroups are caused different sets of risk factors. The primary impetus to establish the AMBER consortium was to pool four large studies to reach an adequate AA sample size to investigate risk factors with breast cancer subtypes. In analyses of pooled data from this large consortium, we have demonstrated etiological heterogeneity by ER status in AA women. ${ }^{32-34}$ Therefore, in this study, we also investigated vitamin D-related genetic variants with breast cancer risk by ER status, as well as ER- vs. ER+ status in case-only analysis. 
The significant pathway-based findings are consistent with our hypothesis that vitamin D signaling and metabolism could be related to aggressive breast cancer in AA women. This is supported by results from our previous study of circulating 25OHD levels and breast cancer aggressive characteristics. In that study, we found that high 25OHD levels were associated with lower risk of ER- cancer, with little influence on ER+ disease. ${ }^{13}$ These converging data from circulating vitamin D biomarkers, genetic variations and reported work on tumor expression, support the notion that active vitamin D signaling may be protective against aggressive breast cancer subtypes. ${ }^{10}$ Similar findings were also reported from other observational studies in breast cancer and prostate cancer, ${ }^{35-39}$ and some experimental studies have provided biological mechanisms underlying the associations with more aggressive cancers. ${ }^{40-42}$ However, there are also studies reporting no associations of vitamin D levels with aggressive cancer characteristics. ${ }^{43}$

The above pathway-level association with ER status was driven by a single gene, $C A S R$, which is crucial to calcium homeostasis. There is evidence of CASR functioning as a tumor suppressor in mammary cells, ${ }^{44}$ and $C A S R$ genetic variations have been related to lethal prostate cancer in EA men. ${ }^{45}$ In another study in Chinese women, rs17251221 in CASR was associated with breast cancer ER status and prognosis. ${ }^{46}$ However, this SNP was not associated with ER status in our study, nor in strong LD with the two significant SNPs in AA population. Because vitamin D is intricately related to calcium homeostasis, it remains a challenge to separate the effects of vitamin D from calcium on cancer.

In an effort to determine whether the associations observed in our study are race-specific or are also present in EA women, we looked up the top variants from Table 3 in a large European population using the GAME-ON GWAS look up tool (http:// gameon.dfci.harvard.edu). We were able to obtain results for 3 out of 12 variants and for a proxy marker in high $\mathrm{LD}\left(r^{2}=0.847\right.$ in Europeans) with a fourth variant in Table 3 . The findings, along with allele frequencies in the European population ( $1 \mathrm{~K}$ Genome $\mathrm{CEU})$, are summarized in Supporting Information Table 1. None of these four variants were associated with either overall or ER- breast cancer risk in Europeans. Another three variants were monomorphic or had very low allele frequency (0.01) in Europeans. For the other five variants in Table 3, we could not obtain association results from the GAME-ON GWAS data for the variants or their proxies. Therefore, we looked up all the variants in the corresponding genes in the GAME-ON GWAS data. Again, no similar associations with breast cancer overall or ER- cancer could be found from this large European population. The lack of consistency of the associations in AA and EA women is not unexpected. In the WCHS, we previously found that genetic associations in several biological pathways, ${ }^{47-50}$ including several vitamin D-related genes, ${ }^{11}$ differed between AA and EA women. These data suggest that associations between genetic variants in vitamin D-related pathways and breast cancer risk in AAs may be different from those in EAs.

In conclusion, in one of the largest breast cancer studies of AA women, we found evidence of associations of vitamin D-related genetic variations with breast cancer risk, particularly with ER- breast cancer. Our finding of $C A S R$ variants associated with tumor ER status suggests a potential role of vitamin D in modulating breast cancer phenotypes and highlights 
the importance of considering tumor heterogeneity in future studies of vitamin D and breast cancer etiology.

\section{Supplementary Material}

Refer to Web version on PubMed Central for supplementary material.

\section{Acknowledgements}

The authors thank participants and staff of the contributing studies. They wish also to acknowledge the late Robert Millikan, DVM, MPH, PhD, who was instrumental in the creation of this consortium. Pathology data were obtained from numerous state cancer registries (Arizona, California, Colorado, Connecticut, Delaware, District of Columbia, Florida, Georgia, Hawaii, Illinois, Indiana, Kentucky, Louisiana, Maryland, Massachusetts, Michigan, New Jersey, New York, North Carolina, Oklahoma, Pennsylvania, South Carolina, Tennessee, Texas, Virginia), and the results do not necessarily represent their views.

Grant sponsor: National Cancer Institute; Grant numbers: P01CA151135, R01CA058420, UM1CA164974, R01CA098663, R01CA100598, U19 CA1480655; Grant sponsor: University Cancer Research Fund of North Carolina; Grant sponsor: Breast Cancer Research Foundation

\section{References}

1. Deeb KK, Trump DL, Johnson CS. Vitamin D signalling pathways in cancer: potential for anticancer therapeutics. Nat Rev Cancer. 2007; 7:684-700. [PubMed: 17721433]

2. Feldman D, Krishnan AV, Swami S, et al. The role of vitamin D in reducing cancer risk and progression. Nat Rev Cancer. 2014; 14:342-57. [PubMed: 24705652]

3. Grant WB. A multicountry ecological study of cancer incidence rates in 2008 with respect to various risk-modifying factors. Nutrients. 2014; 6:163-89.

4. Kim Y, Je Y. Vitamin D intake, blood 25(OH)D levels, and breast cancer risk or mortality: a metaanalysis. Br J Cancer. 2014; 110:2772-84. [PubMed: 24714744]

5. Chung M, Lee J, Terasawa T, et al. Vitamin D with or without calcium supplementation for prevention of cancer and fractures: an updated meta-analysis for the U.S. Preventive Services Task Force. Ann Intern Med. 2011; 155:827-38. [PubMed: 22184690]

6. Chlebowski RT. Vitamin D and breast cancer: interpreting current evidence. Breast Cancer Res. 2011; 13:217. [PubMed: 21884640]

7. Mun MJ, Kim TH, Hwang JY, et al. Vitamin D receptor gene polymorphisms and the risk for female reproductive cancers: a meta-analysis. Maturitas. 2015; 81:256-65. [PubMed: 25882760]

8. Raimondi S, Johansson H, Maisonneuve P, et al. Review and meta-analysis on vitamin D receptor polymorphisms and cancer risk. Carcinogenesis. 2009; 30:1170-80. [PubMed: 19403841]

9. Zhang K, Song L. Association between vitamin D receptor gene polymorphisms and breast cancer risk: a meta-analysis of 39 studies. PLoS One. 2014; 9:e96125. [PubMed: 24769568]

10. Yao S, Ambrosone CB. Associations between vitamin D deficiency and risk of aggressive breast cancer in African-American women. J Steroid Biochem Mol Biol. 2013; 136:337-41. [PubMed: 22995734]

11. Yao S, Zirpoli G, Bovbjerg DH, et al. Variants in the vitamin D pathway, serum levels of vitamin $\mathrm{D}$, and estrogen receptor negative breast cancer among African-American women: a case-control study. Breast Cancer Res. 2012; 14:R58. [PubMed: 22480149]

12. Anderson WF, Rosenberg PS, Prat A, Perou CM, Sherman ME. How many etiological subtypes of breast cancer: two, three, four, or more? J Natl Cancer Inst. 2014; 106:pii: dju 165.

13. Yao S, Sucheston LE, Millen AE, et al. Pretreatment serum concentrations of 25-hydroxyvitamin D and breast cancer prognostic characteristics: a case-control and a case-series study. PLoS One. 2011; 6:e17251. [PubMed: 21386992] 
14. Palmer JR, Ambrosone CB, Olshan AF. A collaborative study of the etiology of breast cancer subtypes in African American women: the AMBER consortium. Cancer Causes Control. 2014; 25:309-19. [PubMed: 24343304]

15. Newman B, Moorman PG, Millikan R, et al. The Carolina Breast Cancer Study: integrating population-based epidemiology and molecular biology. Breast Cancer Res Treat. 1995; 35:51-60. [PubMed: 7612904]

16. Ambrosone CB, Ciupak GL, Bandera EV, et al. Conducting molecular epidemiological research in the age of HIPAA: a multi-institutional case-control study of breast cancer in African-American and European-American Women. J Oncol. 2009; 2009:871250. [PubMed: 19865486]

17. Bandera EV, Chandran U, Zirpoli G, et al. Rethinking sources of representative controls for the conduct of case-control studies in minority populations. BMC Med Res Methodol. 2013; 13:71. [PubMed: 23721229]

18. Rosenberg L, Adams-Campbell L, Palmer JR. The Black Women's Health Study: a follow-up study for causes and preventions of illness. J Am Med Womens Assoc. 1995; 50:56-8. [PubMed: 7722208]

19. Kolonel LN, Henderson BE, Hankin JH, et al. A multiethnic cohort in Hawaii and Los Angeles: baseline characteristics. Am J Epidemiol. 2000; 151:346-57. [PubMed: 10695593]

20. Subramanian A, Tamayo P, Mootha VK, et al. Gene set enrichment analysis: a knowledge-based approach for interpreting genome-wide expression profiles. Proc Natl Acad Sci USA. 2005; 102:15545-50. [PubMed: 16199517]

21. Abecasis GR, Auton A, Brooks LD, et al. An integrated map of genetic variation from 1,092 human genomes. Nature. 2012; 491:56-65. [PubMed: 23128226]

22. Laurie CC, Doheny KF, Mirel DB, et al. Quality control and quality assurance in genotypic data for genome-wide association studies. Genet Epidemiol. 2010; 34:591-602. [PubMed: 20718045]

23. Howie BN, Donnelly P, Marchini J. A flexible and accurate genotype imputation method for the next generation of genome-wide association studies. PLoS Genet. 2009; 5:e1000529. [PubMed: 19543373]

24. Price AL, Patterson NJ, Plenge RM, et al. Principal components analysis corrects for stratification in genome-wide association studies. Nat Genet. 2006; 38:904-9. [PubMed: 16862161]

25. Purcell S, Neale B, Todd-Brown K, et al. PLINK: a tool set for whole-genome association and population-based linkage analyses. Am J Hum Genet. 2007; 81:559-75. [PubMed: 17701901]

26. Yu K, Li Q, Bergen AW, et al. Pathway analysis by adaptive combination of P-values. Genet Epidemiol. 2009; 33:700-9. [PubMed: 19333968]

27. Gao X. Multiple testing corrections for imputed SNPs. Genet Epidemiol. 2011; 35:154-8. [PubMed: 21254223]

28. Pruim RJ, Welch RP, Sanna S, et al. LocusZoom: regional visualization of genome-wide association scan results. Bioinformatics. 2010; 26:2336-7. [PubMed: 20634204]

29. Ahn J, Yu K, Stolzenberg-Solomon R, et al. Genome-wide association study of circulating vitamin D levels. Hum Mol Genet. 2010; 19:2739-45. [PubMed: 20418485]

30. Wang TJ, Zhang F, Richards JB, et al. Common genetic determinants of vitamin D insufficiency: a genome-wide association study. Lancet. 2010; 376:180-8. [PubMed: 20541252]

31. Mondul AM, Shui IM, Yu K, et al. Vitamin D-associated genetic variation and risk of breast cancer in the breast and prostate cancer cohort consortium (BPC3). Cancer Epidemiol Biomarkers Prev. 2015; 24:627-30. [PubMed: 25542828]

32. Ambrosone CB, Zirpoli G, Hong CC, et al. Important role of menarche in development of estrogen receptor-negative breast cancer in African American Women. J Natl Cancer Inst. 2015; 107:pii: djv172.

33. Palmer JR, Viscidi E, Troester MA, et al. Parity, lactation, and breast cancer subtypes in African American women: results from the AMBER Consortium. J Natl Cancer Inst. 2014; 106:pii: dju237.

34. Rosenberg L, Bethea TN, Viscidi E, et al. Post-menopausal female hormone use and risk of estrogen receptor positive and negative breast cancer in African American women. J Natl Cancer Inst. 2015; 108:pii: djv361. 
35. Hatse S, Lambrechts D, Verstuyf A, et al. Vita-min D status at breast cancer diagnosis: correlation with tumor characteristics, disease outcome, and genetic determinants of vitamin D insufficiency. Carcinogenesis. 2012; 33:1319-26. [PubMed: 22623648]

36. Tretli S, Schwartz GG, Torjesen PA, et al. Serum levels of 25-hydroxyvitamin D and survival in Norwegian patients with cancer of breast, colon, lung, and lymphoma: a population-based study. Cancer Causes Control. 2012; 23:363-70. [PubMed: 22193397]

37. Villasenor A, Ballard-Barbash R, Ambs A, et al. Associations of serum 25-hydroxyvitamin D with overall and breast cancer-specific mortality in a multiethnic cohort of breast cancer survivors. Cancer Causes Control. 2013; 24:759-67. [PubMed: 23361338]

38. Vrieling A, Hein R, Abbas S, et al. Serum 25-hydroxyvitamin D and postmenopausal breast cancer survival: a prospective patient cohort study. Breast Cancer Res. 2011; 13:R74. [PubMed: 21791049]

39. Shui IM, Mucci LA, Kraft P, et al. Vitamin D-related genetic variation, plasma vitamin D, and risk of lethal prostate cancer: a prospective nested case-control study. J Natl Cancer Inst. 2012; 104:690. [PubMed: 22499501]

40. Pendas-Franco N, Gonzalez-Sancho JM, Suarez Y, et al. Vitamin D regulates the phenotype of human breast cancer cells. Differentiation. 2007; 75:193-207. [PubMed: 17288543]

41. Zhang Y, Leung DY, Richers BN, et al. Vitamin D inhibits monocyte/macrophage proinflammatory cytokine production by targeting MAPK phosphatase-1. J Immunol. 2012; 188:2127-35. [PubMed: 22301548]

42. Pervin S, Hewison M, Braga M, et al. Down-regulation of vitamin D receptor in mammospheres: implications for vitamin D resistance in breast cancer and potential for combination therapy. PLoS One. 2013; 8:e53287. [PubMed: 23341935]

43. Eliassen AH, Spiegelman D, Hollis BW, et al. Plasma 25-hydroxyvitamin D and risk of breast cancer in the Nurses' Health Study II. Breast Cancer Res. 2011; 13:R50. [PubMed: 21569367]

44. Brennan SC, Thiem U, Roth S, et al. Calcium sensing receptor signalling in physiology and cancer. Biochim Biophys Acta. 2013; 1833:1732-44. [PubMed: 23267858]

45. Shui IM, Mucci LA, Wilson KM, et al. Common genetic variation of the calcium-sensing receptor and lethal prostate cancer risk. Cancer Epidemiol Biomarkers Prev. 2013; 22:118-26. [PubMed: 23125333]

46. Li X, Kong X, Jiang L, et al. A genetic polymorphism (rs17251221) in the calcium-sensing receptor is associated with breast cancer susceptibility and prognosis. Cell Physiol Biochem. 2014; 33:165-72. [PubMed: 24481145]

47. Gong Z, Quan L, Yao S, et al. Innate immunity pathways and breast cancer Risk in African American and European-American women in the Women's Circle of Health Study (WCHS). PLoS One. 2013; 8:e72619. [PubMed: 23991131]

48. Quan L, Gong Z, Yao S, et al. Cytokine and cytokine receptor genes of the adaptive immune response are differentially associated with breast cancer risk in American women of African and European ancestry. Int J Cancer. 2014; 134:1408-21. [PubMed: 23996684]

49. Quan L, Hong CC, Zirpoli G, et al. Variants of estrogen-related genes and breast cancer risk in European and African American women. Endocr Relat Cancer. 2014; 21:853-64. [PubMed: 25228414]

50. Yao S, Graham K, Shen J, et al. Genetic variants in microRNAs and breast cancer risk in African American and European American women. Breast Cancer Res Treat. 2013; 141:447-59. [PubMed: 24062209] 


\section{What's new?}

Vitamin D displays many anti-cancer activities. While African Americans are known to have a high prevalence of vitamin D deficiency, studies of genetic variations in vitamin D-related pathways and breast cancer risk have been conducted mostly in populations of European ancestry. This study is the largest and most comprehensive investigation of vitamin D-related genetic variations with breast cancer risk and tumor estrogen receptor status in African American women. The data reveal modest associations of genetic variations in vitamin D pathways with breast cancer risk, and suggest a role for vitamin D in risk of estrogen receptor negative breast cancer. 

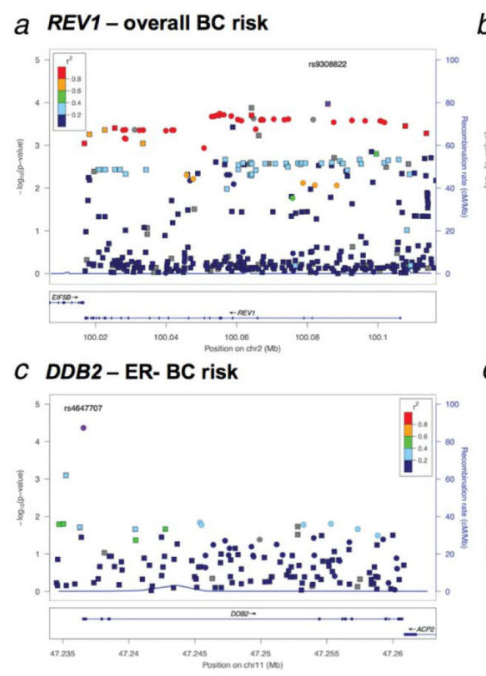

b ERCC6- $\mathrm{ER}+\mathrm{BC}$ risk

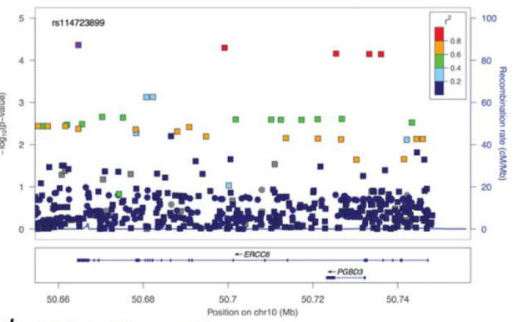

d CASR-ER-vS. ER+

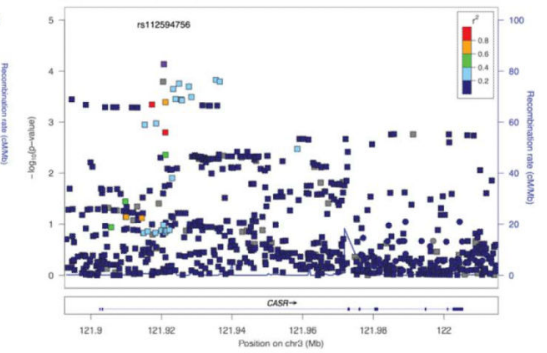

Figure 1.

Plots of log-transformed $p$-values from single marker analysis for top genes in each subgroup test were generated using the Locus-Zoom program. The labeled marker in the plots were the most significant SNP (index SNP) in each gene, and the LD between the each of other markers in the gene and the index SNP was color coded, with red color indicating strong LD $\left(r^{2}>0.8\right)$ and blue color indicating weak LD $\left(t^{2}<0.2\right)$. Genotyped SNPs were indicated by closed dots and imputed SNPs were indicated by closed squares. 


\section{Table 1}

Descriptive characteristics of the study populations in the AMBER consortium

\begin{tabular}{|c|c|c|c|c|c|c|c|c|}
\hline & \multicolumn{2}{|l|}{$\underline{\text { BWHS }}$} & \multicolumn{2}{|l|}{$\underline{\text { CBCS }}$} & \multicolumn{2}{|l|}{ MEC } & \multicolumn{2}{|l|}{$\underline{\text { WCHS }}$} \\
\hline & $\begin{array}{l}\text { Case, } \\
n(\%)\end{array}$ & $\begin{array}{l}\text { Control, } \\
n(\%)\end{array}$ & $\begin{array}{l}\text { Case, } \\
n(\%)\end{array}$ & $\begin{array}{l}\text { Control, } \\
n(\%)\end{array}$ & $\begin{array}{l}\text { Case, } \\
n(\%)\end{array}$ & $\begin{array}{l}\text { Control, } \\
n(\%)\end{array}$ & $\begin{array}{l}\text { Case, } \\
n(\%)\end{array}$ & $\begin{array}{l}\text { Control, } \\
n(\%)\end{array}$ \\
\hline Total & 901 & 2249 & 1408 & 615 & 533 & 989 & 821 & 834 \\
\hline \multicolumn{9}{|l|}{ Age group } \\
\hline $18-39$ & $47(5)$ & $217(9)$ & $204(14)$ & $87(14)$ & $0(0)$ & $0(0)$ & $85(10)$ & $116(14)$ \\
\hline $40-49$ & $262(29)$ & $652(29)$ & $459(33)$ & $211(34)$ & $9(2)$ & $16(2)$ & $215(26)$ & $228(27)$ \\
\hline $50-59$ & $302(34)$ & $770(34)$ & $381(27)$ & $150(24)$ & $112(21)$ & $222(22)$ & $292(36)$ & $319(38)$ \\
\hline $60-69$ & $204(23)$ & $442(20)$ & $267(19)$ & $114(19)$ & $175(33)$ & $288(29)$ & $173(21)$ & $142(17)$ \\
\hline $70+$ & $86(9)$ & $168(7)$ & $97(7)$ & $53(9)$ & $237(44)$ & $463(47)$ & $56(7)$ & $29(3)$ \\
\hline \multicolumn{9}{|c|}{ Estrogen receptor status in cases } \\
\hline Positive & $498(55)$ & - & $741(53)$ & - & $309(58)$ & - & $435(53)$ & - \\
\hline Negative & $233(26)$ & - & $565(40)$ & - & $135(25)$ & - & $165(20)$ & - \\
\hline Unknown & $170(19)$ & - & $102(7)$ & - & $89(17)$ & - & $221(27)$ & - \\
\hline
\end{tabular}


Table 2

$p$-values of pathway- and gene-level test with breast cancer risk

\begin{tabular}{|c|c|c|c|c|c|c|}
\hline Gene & \# Total Marker & \# Effective Marker & Overall & ER+ & ER- & ER-vs. ER+ \\
\hline Pigment synthesis and metabolism & 13,598 & 5,227 & 0.99 & 0.74 & 0.15 & 0.16 \\
\hline$A L A D$ & 181 & 115 & 0.21 & 0.68 & 0.29 & 0.58 \\
\hline$A L A S 1$ & 91 & 52 & 0.49 & 0.67 & 0.59 & 0.39 \\
\hline$A L A S 2$ & 92 & 51 & 0.66 & 0.07 & 0.53 & 0.05 \\
\hline$A P 3 D 1$ & 433 & 144 & 0.46 & 0.84 & 0.96 & 0.94 \\
\hline$A S I P$ & 124 & 46 & 0.72 & 0.70 & 0.31 & 0.37 \\
\hline$B L V R A$ & 267 & 100 & 0.51 & 0.36 & 0.34 & 0.28 \\
\hline COX10 & 744 & 296 & 0.87 & 0.88 & 0.98 & 0.76 \\
\hline COX15 & 93 & 42 & 0.94 & 0.61 & 0.95 & 0.24 \\
\hline CPOX & 231 & 113 & 0.43 & 0.84 & 0.59 & 0.78 \\
\hline$D C T$ & 372 & 139 & 0.34 & 0.64 & 0.003 & 0.01 \\
\hline EXOC2 & 1555 & 705 & 0.19 & 0.21 & 0.35 & 0.51 \\
\hline$F E C H$ & 359 & 179 & 0.71 & 0.68 & 0.25 & 0.23 \\
\hline GMPS & 410 & 82 & 0.68 & 0.17 & 0.56 & 0.29 \\
\hline GPR143 & 293 & 137 & 0.14 & 0.14 & 0.90 & 0.78 \\
\hline HERC2 & 1133 & 285 & 0.48 & 0.40 & 0.72 & 0.61 \\
\hline IRF4 & 256 & 160 & 0.37 & 0.65 & 0.07 & 0.007 \\
\hline$K I T L G$ & 369 & 90 & 0.95 & 1.00 & 0.49 & 0.56 \\
\hline$M C 1 R$ & 38 & 28 & 0.38 & 0.49 & 0.97 & 0.62 \\
\hline NFE2L1 & 129 & 62 & 0.97 & 0.97 & 0.60 & 0.73 \\
\hline$O C A 2$ & 2511 & 786 & 0.67 & 0.76 & 0.38 & 0.29 \\
\hline PAICS & 158 & 41 & 0.27 & 0.03 & 0.43 & 0.23 \\
\hline PPOX & 57 & 33 & 0.24 & 0.73 & 0.50 & 0.21 \\
\hline$S L C 24 A 4$ & 1642 & 801 & 0.18 & 0.47 & 0.04 & 0.09 \\
\hline$S L C 24 A 5$ & 69 & 32 & 0.47 & 0.88 & 0.15 & 0.23 \\
\hline SLC45A2 & 244 & 157 & 0.55 & 0.32 & 0.87 & 0.31 \\
\hline TPCN2 & 417 & 143 & 0.48 & 0.26 & 0.83 & 0.31 \\
\hline$T S P O$ & 175 & 95 & 0.83 & 0.46 & 0.27 & 0.34 \\
\hline$T Y R$ & 933 & 240 & 0.68 & 0.76 & 0.97 & 0.57 \\
\hline$T Y R P 1$ & 222 & 73 & 0.78 & 0.79 & 0.55 & 0.87 \\
\hline Response to UV exposure & 5,814 & 1,886 & 0.13 & 0.09 & 0.80 & 0.71 \\
\hline$B C L 3$ & 214 & 131 & 0.03 & 0.01 & 0.22 & 0.45 \\
\hline BRSK1 & 175 & 114 & 0.93 & 0.86 & 0.99 & 0.99 \\
\hline$C D K N 2 D$ & 26 & 14 & 0.77 & 0.44 & 0.81 & 0.18 \\
\hline$D D B 2$ & 160 & 74 & 0.19 & 0.92 & 0.05 & 0.03 \\
\hline ERCC2 & 139 & 82 & 0.19 & 0.65 & 0.52 & 0.70 \\
\hline ERCC3 & 248 & 78 & 0.74 & 0.75 & 0.81 & 0.89 \\
\hline ERCC4 & 332 & 107 & 0.99 & 1.00 & 0.25 & 0.58 \\
\hline ERCC5 & 187 & 80 & 0.52 & 0.68 & 0.94 & 0.60 \\
\hline
\end{tabular}




\begin{tabular}{|c|c|c|c|c|c|c|}
\hline Gene & \# Total Marker & \# Effective Marker & Overall & ER+ & ER- & ER-vs. ER+ \\
\hline ERCC6 & 493 & 108 & 0.23 & 0.01 & 0.88 & 0.22 \\
\hline ERCC8 & 414 & 87 & 0.49 & 0.64 & 0.33 & 0.66 \\
\hline FEN1 & 13 & 10 & 0.86 & 0.93 & 0.29 & 0.63 \\
\hline$G P X 1$ & 51 & 24 & 0.64 & 0.52 & 0.95 & 0.95 \\
\hline IL $12 \mathrm{~A}$ & 5 & 5 & 0.73 & 0.65 & 0.40 & 0.49 \\
\hline$I L 12 B$ & 106 & 55 & 0.82 & 0.68 & 0.99 & 0.85 \\
\hline$I V L$ & 197 & 90 & 0.39 & 0.52 & 0.86 & 0.96 \\
\hline МАРК 8 & 763 & 122 & 0.05 & 0.10 & 0.21 & 0.35 \\
\hline POLD1 & 355 & 99 & 0.35 & 0.37 & 0.73 & 0.81 \\
\hline$R E L A$ & 94 & 44 & 0.20 & 0.11 & 0.27 & 0.13 \\
\hline$R E V 1$ & 391 & 90 & 0.004 & 0.02 & 0.22 & 0.52 \\
\hline RPAIN & 71 & 26 & 0.82 & 0.79 & 0.85 & 0.80 \\
\hline SCARA3 & 384 & 146 & 0.31 & 0.34 & 0.79 & 0.37 \\
\hline SERPINB13 & 199 & 79 & 0.11 & 0.15 & 0.88 & 1.00 \\
\hline$U B E 4 B$ & 797 & 221 & 0.50 & 0.70 & 0.62 & 0.39 \\
\hline Vitamin D metabolism and signaling & 5,033 & 2,127 & 0.54 & 0.45 & 0.64 & 0.02 \\
\hline CASR & 755 & 196 & 0.70 & 0.24 & 0.11 & 0.001 \\
\hline CYP24A1 & 323 & 178 & 0.44 & 0.61 & 0.84 & 0.83 \\
\hline CYP27A1 & 245 & 92 & 0.31 & 0.44 & 0.58 & 0.64 \\
\hline$C Y P 27 B 1$ & 15 & 14 & 0.81 & 0.99 & 0.21 & 0.54 \\
\hline$C Y P 2 R 1$ & 63 & 43 & 0.05 & 0.04 & 0.43 & 0.32 \\
\hline$G C$ & 450 & 178 & 0.23 & 0.35 & 0.55 & 0.49 \\
\hline NCOA1 & 802 & 182 & 0.89 & 0.89 & 0.56 & 0.86 \\
\hline$R X R A$ & 1004 & 464 & 0.70 & 0.47 & 0.43 & 0.16 \\
\hline$R X R B$ & 35 & 25 & 0.37 & 0.16 & 0.86 & 0.91 \\
\hline$S M A D 3$ & 837 & 448 & 0.12 & 0.21 & 0.10 & 0.38 \\
\hline$V D R$ & 504 & 307 & 0.41 & 0.43 & 0.47 & 0.77 \\
\hline
\end{tabular}

For the columns "Overall", "ER+" and "ER-", the tests were comparing breast cancer as a single entity, ER+ cancer, and ER- cancer with healthy controls, respectively. 
Table 3

Top markers associated with breast cancer risk with a corrected $p$ values $₫ 0.05$

\begin{tabular}{|c|c|c|c|c|c|c|c|c|}
\hline SNP & Gene & Function & A1/A2 & $\begin{array}{l}\text { A1 } \\
\text { frequency }\end{array}$ & $\begin{array}{l}\text { Info } \\
\text { score }\end{array}$ & OR $(95 \% \mathrm{CI})$ & $p$ & $\begin{array}{l}\text { Corrected } \\
p\end{array}$ \\
\hline \multicolumn{9}{|c|}{ Overall breast cancer risk } \\
\hline rs9308822 & $R E V 1$ & intronic & $\mathrm{C} / \mathrm{T}$ & 0.27 & 1.00 & $0.86(0.80-0.93)$ & $1.1 \mathrm{E}-04$ & 0.01 \\
\hline rs34698726 & $B C L 3$ & intergenic & $\mathrm{T} / \mathrm{A}$ & 0.32 & 0.91 & $1.16(1.07-1.25)$ & $1.3 \mathrm{E}-04$ & 0.02 \\
\hline rs 10700835 & PAICS & intergenic & $\mathrm{ACT} / \mathrm{A}$ & 0.51 & 1.01 & $1.12(1.05-1.20)$ & $1.0 \mathrm{E}-03$ & 0.04 \\
\hline \multicolumn{9}{|c|}{ ER-positive breast cancer } \\
\hline rs114723899 & ERCC6 & $3^{\prime}$ UTR & $\mathrm{C} / \mathrm{T}$ & 0.04 & 0.95 & $0.62(0.49-0.78)$ & $4.3 \mathrm{E}-05$ & 0.005 \\
\hline rs 10700835 & PAICS & intergenic & $\mathrm{ACT} / \mathrm{A}$ & 0.51 & 1.01 & $1.15(1.07-1.25)$ & 4.4E-04 & 0.02 \\
\hline rs190770932 & CYP2R1 & intronic & $\mathrm{A} / \mathrm{G}$ & 0.03 & 0.94 & $1.55(1.21-1.99)$ & $4.8 \mathrm{E}-04$ & 0.02 \\
\hline rs34698726 & $B C L 3$ & intergenic & $\mathrm{T} / \mathrm{A}$ & 0.32 & 0.92 & $1.19(1.09-1.30)$ & $1.7 \mathrm{E}-04$ & 0.02 \\
\hline rs 13431410 & $R E V 1$ & intergenic & $\mathrm{A} / \mathrm{G}$ & 0.26 & 0.97 & $0.84(0.77-0.93)$ & $4.8 \mathrm{E}-04$ & 0.04 \\
\hline \multicolumn{9}{|c|}{ ER-negative breast cancer } \\
\hline rs4647707 & $D D B 2$ & $5^{\prime}$ UTR & $\mathrm{A} / \mathrm{G}$ & 0.32 & 1.02 & $1.26(1.13-1.41)$ & $4.3 \mathrm{E}-05$ & 0.003 \\
\hline rs112907967 & $D C T$ & intronic & $\mathrm{T} / \mathrm{C}$ & 0.11 & 1.00 & $1.34(1.14-1.57)$ & $3.0 \mathrm{E}-04$ & 0.04 \\
\hline \multicolumn{9}{|c|}{$\begin{array}{l}\text { ER-negative vs. positive breast } \\
\text { cancer }\end{array}$} \\
\hline rs112594756 & CASR & intronic & $\mathrm{G} / \mathrm{C}$ & 0.34 & 0.89 & $1.27(1.13-1.43)$ & $7.3 \mathrm{E}-05$ & 0.01 \\
\hline rs6799828 & CASR & intronic & $\mathrm{G} / \mathrm{T}$ & 0.56 & 0.94 & $1.24(1.11-1.39)$ & $1.5 \mathrm{E}-04$ & 0.03 \\
\hline rs3837536 & $D C T$ & intronic & G/GA & 0.60 & 1.00 & $1.23(1.11-1.38)$ & $1.8 \mathrm{E}-04$ & 0.03 \\
\hline
\end{tabular}

Footnote: A1 and A2 indicate risk and reference alleles, respectively. $p$ values corrected for multiple comparison using simpleM approach. OR, odds ratio for the risk allele. CI, confidence interval. 